\title{
A Millimeter-wave Radar Signal Processing Method based on FPGA+DSP
}

\author{
Dejun Chen, Yang Liu, Yu Yin, Dong Liu \\ School of Information Engineering, Wuhan University of Technology, Wuhan, China
}

Keywords: millimeter-wave radar; FPGA+DSP; signal processing

\begin{abstract}
By analyzing basic requirements and characters of millimeter-wave radar signals, this paper proposed signal processing methods using Linear Frequency Modulated Continuous Wave based on FPGA+DSP. FPGA controls peripheral ADC and DAC to complete modulation triangular wave generation and radar Intermediate frequency (IF) signal sample. Floating-point DSP helps to complete the digital signal filtering and spectral analysis, and to detect target's speed and orientation information from the real-time analysis of the radar IF signal. Both the principle and computer simulation of the method are demonstrated in detail.
\end{abstract}

\section{Introduction}

With the popularity of the family car, attendant problems like traffic safety is threatening the life and property safety of the public, hence makes the study of vehicle safety increasingly becoming the focus of attention. Millimeter-wave radar has merits of small size, low power consumption, moderate detection ranges, which meet the needs of vehicle equipment. Active detection of external environment by millimeter-wave gains speed and orientation information of surrounding road vehicles, combining it with the related early warning algorithm can effectively reduce collision accidents.

Millimeter-wave radar application needs a supportive signal processing system, which is limited by vehicle environment such as system size and power consumption. As vehicle has complex travelling environment, lots of outside interference, whether the radar signal processing method can accurately extract the speed and orientation information of the target is tightly related to whether the warning is accurate or not ${ }^{[3]}$, hence it is essential to study this method. In this respect, Germany, Japan and the United States has begun related research much earlier, some products they invented have already been used in the market. While because of lacking core components and technology for radar systems, researches in China are still developed in universities and labs. Radar signal processing system based on DSP is very common now, but drive capability of DSP peripheral required by sampling and signal generation in system is very high, which will also affect the processing capability of the system. The current research literature on this issue is rare.

Based on the requirements above, this paper designs a FPGA + DSP millimeter-wave radar signal processing system. By combining the powerful hardware, which contains FPGA's strong peripheral drive capability and DSP 's robust data processing capability, with signal filtering and spectral analysis, this system achieves the purpose of extracting accurate radar target information contained in the IF signal.

\section{Principles of Millimeter-wave Radar}

(1)Speed and distance measurement principles

Speed and distance measurement principles of millimeter-wave radar are to firstly generate high-frequency transmission signal with frequency changing in linear times by periodic triangular wave modulation. Then when it meets an obstacle, the receiver antenna will receive its reflection. Finally, after a series of RF front-end processing, the system outputs radar IF signal contained target information. Since the amplitude of the triangular wave is divided into two phases of rise and fall, the generated IF signal correspondingly divided into upper and lower sweep frequency. It is 
assumed that the upper and lower sweep frequency are respectively $f_{b^{+}}$and $f_{b^{-}}$, scanning period of transmitted signal is $\mathrm{T}$, the bandwidth is $B$, the center frequency is $f_{0}$, and the electromagnetic wave propagation velocity is the speed of light $c$, hence the speed and distance of the target can be expressed as:

$v=\frac{c\left(f_{b^{-}}-f_{b^{+}}\right)}{4 f_{0}} ; \quad R=\frac{c T\left(f_{b^{-}}+f_{b^{+}}\right)}{8 B}$

(2)Angle measurement principle

Angle measurement is realized by computing the phase differences between two received signals by two independent receiving antennas. Assuming that $\theta$ is the angle we want to get. As the two antennas have a distance $d$, there would be wave path difference $\Delta R$ between the received signals, which would result in a phase difference $\Delta \varphi$. Suppose $\lambda$ is the wavelength of radar, and we could get:

$\theta=\arcsin \left(\frac{\lambda \cdot \Delta \varphi}{2 \pi \cdot d}\right)$

To sum up, millimeter-wave radar could extract the relative speed, distance and angle of the target from radar IF signals, which is all that needs to complete the recognition process.

\section{Hardware Design}

\subsection{Signal generation and sampling circuit}

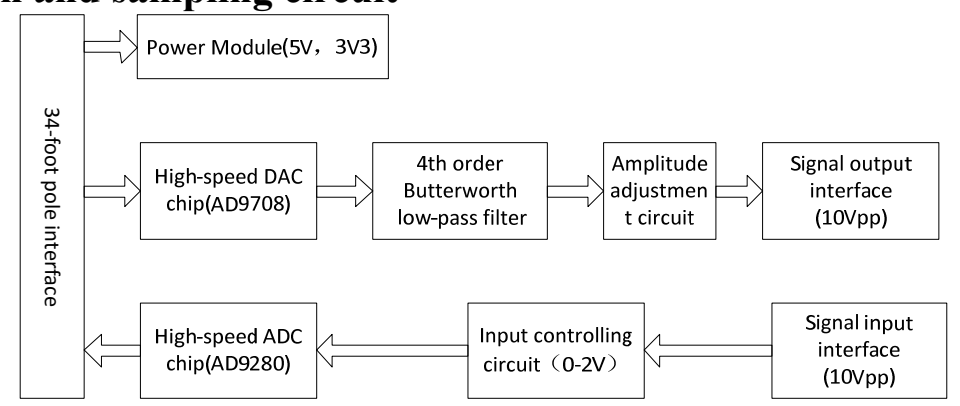

Fig. 1 circuit structure diagram of signal generation and sampling block

Modulated signal generation and radar IF signal sampling block are shown in Fig. 1, both of which is controlled by FPGA timing controller and connected with FPGA through a 34-foot pole interface. D/A and A/D conversion, which are the AD9708 and AD9280, are both 8-bit parallel converter and have 125MSPS converting rate, maximum 32MSPS sampling rate separately. The radar modulation signal is generally about $1 \mathrm{kHz}$, and the IF signal are less then $1 \mathrm{MHz}$, which are in full compliance with the system requirements.

\subsection{Communication between FPGA and DSP}

Because FPGA and DSP have different clocks, in order to ensure the high-speed transmission between this two devices, FPGA use FIFO block as cache to connect with the Xintf bus interface of DSP. The connection diagram is shown in Fig. 2. Connect FPGA's input data bus data[15...0], write clock signal wrclk and write request signal wrreq, which are controlled by sampling block, to the data bus of sampling, sampling clock and sampling synchronization signals, respectively. After sampling points in FIFO are full, we could send data to DSP. Then, write full signal wrfull will produce high level to trigger DSP's external interrupt. DSP read request signal $\overline{\mathrm{XRD}}$ and chip select signals $\overline{\mathrm{XZCS} 0}$ are required to provide the FIFO read request signal rdreq and the clock signal rdclk. In additional, DSP data bus $\mathrm{XD}[15 \ldots 0]$ is connected to the FIFO output bus $\mathrm{q}[15 \ldots 0]$ for parallel transmission of data. 


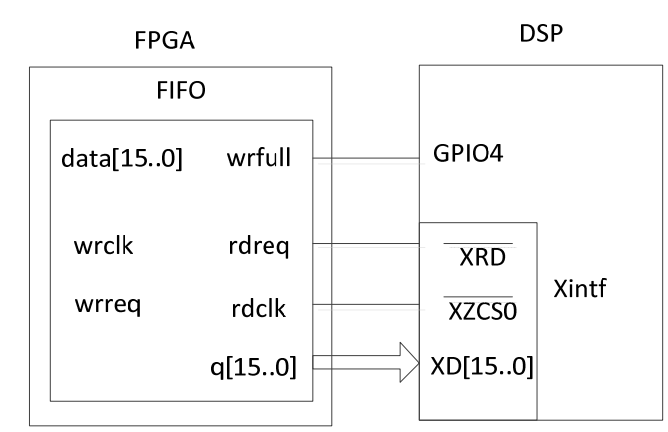

Fig. 2 communication connection between FPGA and DSP

\section{Software Design}

The procedure of radar signal processing is shown in Fig. 3. Procedure before sampling is implemented by timing control of FPGA, and after is realized through analyzing the radar IF signal with digital signal processing algorithms, which mainly contains adaptive filtering algorithm and All-phase FFT (apFFT). Here mainly depicts the implementation of these algorithms on DSP.

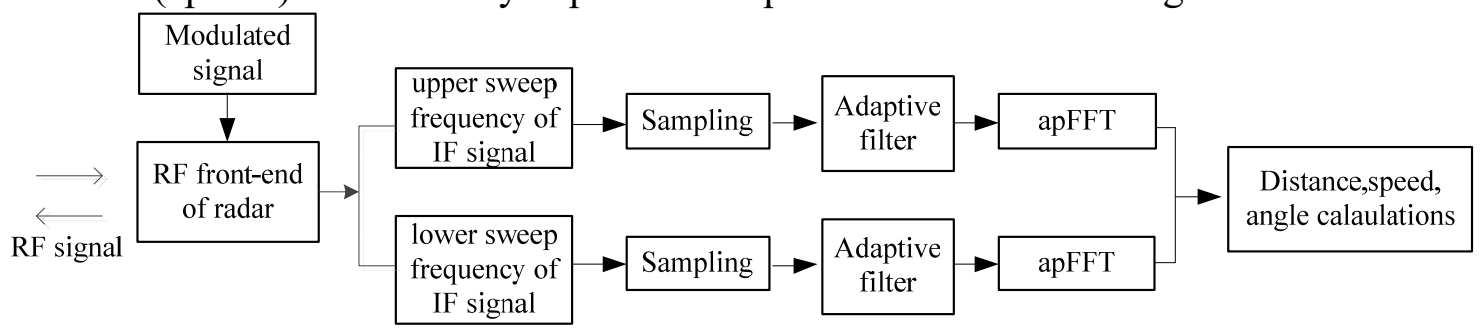

\subsection{Adaptive filer}

Fig. 3 Radar signal processing

Due to the complex driving environment, the filter with fixed parameters can not reach the optimal filter effect in this case. While base on the initial set of weights, the adaptive filtering algorithm can adjust their filter parameters according to the varying of the input signals.

Adaptive filter is composed of two parts: the adaptive filtering algorithms and parameters adjustable digital filter ${ }^{[4]}$. According to different optimization parameters, adaptive algorithm can be divided into Recursive Least Square(RLS) and Least Mean Square(LMS). Though the performance of RLS algorithm is good, its implementation process is complex, and particularly it is difficult to achieve real-time requirements in the automotive environment. In contrast, LMS algorithm is much simple and suitable for mobile platform. Besides, the effect can meet the requirements of radar signal processing, hence we choose LMS adaptive filtering algorithm here. Its implementation on the DSP is shown in Fig. 4.

Where $\mu$ is the step factor which determines the required time to find the best weight vectors of adaptive filter. When the value of $\mu$ is relatively small, the filtering system will be more stable, and the change of the output signal will be relatively flat, but there is also a corresponding increase in adaptive time. Especially when $\mu$ is too small and the number of iterations $i$ are not enough, the system may still not find the optimum filter parameters even if iterations are over. When $\mu$ becomes larger, the signal adaptive process will speed up, but the stability of the system will begin to deteriorate, changes of the output signal will also be larger. If $\mu$ exceeds a critical value, the system output will even become chaos. Considering both effects above, after lots of debugging we select the initial parameters $\mu=0.0026$, the initial weight vector $W[2]=[0,1]$, and the number of iterations $i=1000$. The cycle section of flow chart showing the adaptive procedure which is looking for the best weight vector, where Noise here is the correlation signal of noise, $Y$ is the expectation of noise, $X$ denotes the original signal, and $E$ is the output of the adaptive filter. when iteration is completed, $Y$ will be really close to the noise signal, hence the filtered signal can be obtained through $X-Y$. 


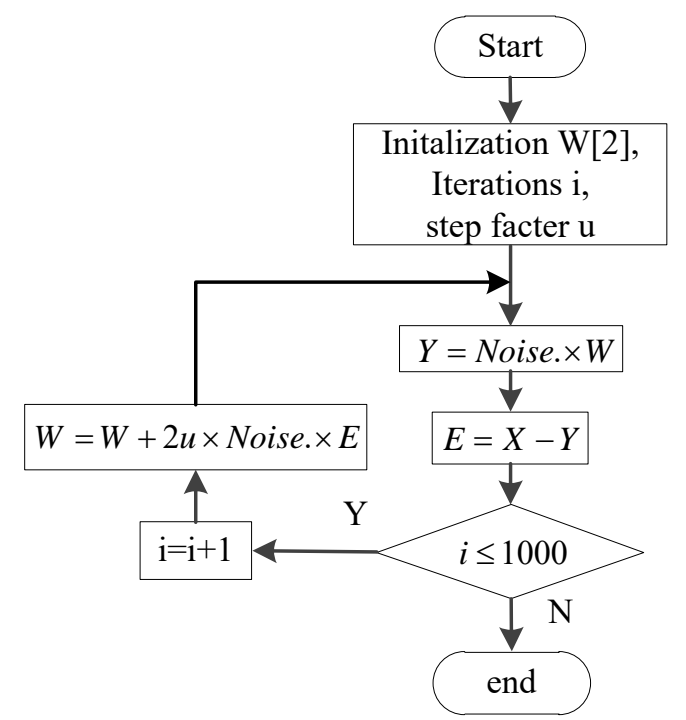

Fig. 4 Program flow chart of adaptive filter

\subsection{All-phase FFT spectrum analysis}

FFT is the most used algorithm in signal spectrum analysis ${ }^{[5]}$, however, because of the spectrum leakage, the precision of the spectrum analysis can be seriously affected ${ }^{[6]}$. Accuracy of radar signal frequency directly affects the ability of the final target recognition, so here we choose all-phase FFT to analyze radar signal spectrums. In contrast to FFT, all-phase FFT has stronger immunity with spectrum leakage, meanwhile it has a phase invariance, which can give accurate phase information with no additional operation ${ }^{[7]}$.

Spectrum analysis procedure of all-phase FFT includes signal preprocessing and FFT. Suppose that a time-domain signal of infinite length has been cut off and then sampled for $2 N-1$ data points. The main idea of signal preprocessing is to divide this $2 N-1$ data points into $N$ groups with $N$ data points for each group. Each group are aligned with the $\mathrm{N}^{\text {th }}$ points, namely the center sample point of $2 N-1$ data points. The remaining data aligned by cyclically shifting. Finally, add the data on corresponding positions and normalize it to get a length of $N$ preprocessing data. Code in C language, which implemented on DSP, is as follows:

$$
\begin{aligned}
& \text { for }(i=0 ; i<2 * N-1 ; i++)\{ \\
& \text { WinAd_data }[\mathrm{i}]=\text { Ad_data }[\mathrm{i}] * \text { hanNing }[\mathrm{i}] \text {; } \\
& \text { for }(\mathrm{i}=0 ; \mathrm{i}<\mathrm{N} ; \mathrm{i}++)\{ \\
& \text { if }(\mathrm{i}==0) \\
& \text { else } \\
& \text { PreAd_data }[\mathrm{i}]=\text { WinAd_data }[\mathrm{N}-1] \text {; } \\
& \text { PreAd_data[i] }=\text { WinAd_data[i-1] }+ \text { WinAd_data[N+i-1]; }
\end{aligned}
$$

The first loop is to add a window to the original data. The implementation of Hanning window function in DSP is that to first design the window function by MATLAB, then export the data to a file and load it in DSP as a header file. The second loop is to divide $2 N-1$ sampling points into two $N$ points groups, then shift the second group and add it with the first one. Processed data can directly compute fast Fourier transformation using FFT library functions provided by TI company. It is important to note that all-phase FFT operation needs to call a large number of data storage space during the calculations. When the DSP internal storage space is insufficient, we could move large arrays to the external storage space by changing the project's CMD file combined with \#pragma DATA_SECTION statement. By applying all-phase FFT, we can get all the frequencies in IF signals. Then extract target speed and orientation from it.

\subsection{Experimental Evaluation}

Mixing the LFMCW modulated by triangular wave, we set IF signal as reference signal, which has center frequency of $76 \mathrm{GHz}$ and modulation period of $10 \mathrm{~ms}$. It's assumed that IF signal contains 
information of three targets: The relative speeds of three targets are $60 \mathrm{~km} / \mathrm{h}, 80 \mathrm{~km} / \mathrm{h}$ and $120 \mathrm{~km} / \mathrm{h}$, respectively. The relative distances are $30 \mathrm{~m}, 60 \mathrm{~m}$ and $120 \mathrm{~m}$, respectively. The relative angles are $-2.87^{\circ}, 2.15^{\circ}$ and $-2.60^{\circ}$, respectively. The whole process of radar signal processing is shown in Fig. 5, where Fig.5(a) denotes sampling result of the upper sweep, Fig.5(b) and Fig.5(c) respectively denote the spectrum before and after filtering. In the figure, the abscissa denotes the sampling point, the ordinate denotes the amplitude of the signal.

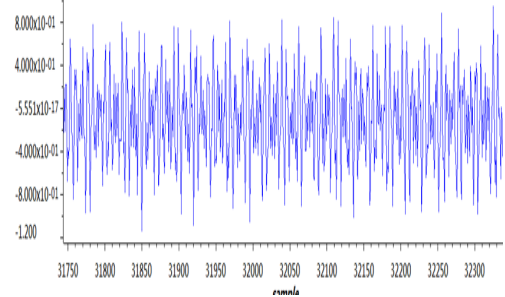

(a)signal sampling result



(b)spectrum without filtering

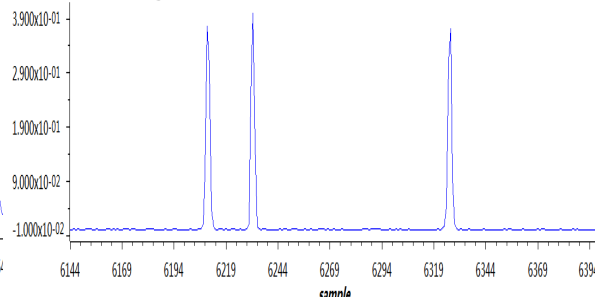

(c)spectrum after filtering

Fig. 5 signal processing

Three peaks in Fig. 6(c), respectively, shows the frequency of $f_{A^{+}}=22916.02 \mathrm{~Hz}$ 、 $f_{B^{+}}=8738.43 \mathrm{~Hz} 、 f_{C^{+}}=1699.63 \mathrm{~Hz}$. Using the same method to lower sweep frequency, we could get another 3 groups of frequency information. By matching its frequency and substituting these frequencies into target measurement formula, here comes the speed, distance and angle results of 3 targets: $\left(120.2 \mathrm{~km} / \mathrm{h}, 120.0 \mathrm{~m},-2.60^{\circ}\right),\left(79.0 \mathrm{~km} / \mathrm{h}, 59.9 \mathrm{~m}, 2.15^{\circ}\right),\left(58.4 \mathrm{~km} / \mathrm{h}, 30.0 \mathrm{~m},-2.87^{\circ}\right)$. Comparison between these three sets of data and experimental data indicates that the errors are within the scope of system performance requirements. It has been tested that a processing period is around $15 \mathrm{~ms}$, which is at the millisecond level, and in line with real-time requirements.

\section{Conclusion}

Millimeter-wave radar is one of the most important means of acquiring surrounding road information in vehicle collision avoidance systems. After adequate processing and analysis of information obtained by IF signals, precise position and status information of the target vehicle could be obtained. This paper presents a FPGA + DSP-based millimeter-wave radar signal processing hardware and software solutions. It adopts embedded hardware, which has small size, low power consumption and is convenient for vehicle environment to achieve. Besides, it improves the accuracy of radar signal processing by adaptive filtering, all-phase pre-processing and other means, at the same time provides a practical solution for the millimeter-wave radar used in automotive collision avoidance system.

\section{Acknowledgment}

This paper has been supported by "the Fundamental Research Funds for the Central Universities (WUT : 2016-zy-044)".

\section{References}

[1] CHEN Yong, HUAGN Xiyue, YANG Shanggang. Research and Development of Automotive Collision Avoidance System[J]. Computer Simulation, 2006, 23(12):239-243.

[2] Song Xiaolin, Feng Guanggang, Yang Jikuang. The Current State and Trends of Automotive Active Collision-avoidance System[J]. Automotive Engineering, 2008, 30(4):285-290.

[3] Ben De, Zhang Gong, Liu Yandong. Magazine Foreword of Radar Signal Processing[J]. Journal of Data Acquisition and Processing, 2014, 29(4):483-485.

[4] Han Haijian, Li Wenhai. Design and performance simulation of the adaptive noise cancellation system[J]. Electronic Measurement Technology, 2015, 38(9):82-85. 
[5] Xu Ming, Liu Wei. An interpolation FFT algorithm based on 8-term cosine window[J]. Power System Protection and Control, 2015, 43(11):27-32.

[6] DIAO Ruipeng, MENG Qingfeng, FAN Hong. Interpolation Algorithms Based on Rife-Vincent Window for Discrete Fourier Transforms of Damped Signals[J]. Journal of Mechanical Engineering, 2015, 51(4):1-7.

[7] WANG Zhaohua, HUANG Xiangdong, YANG Wei. The Measuring Phase Method of All-Phase FFT[J]. WORLD SCI-TECH R\&D, 2007, 29(4):28-32. 